\title{
Emerging strengths in Asia Pacific bioinformatics
} Shoba Ranganathan ${ }^{* 1,2}$, Wen-Lian Hsu ${ }^{3,4}$, Ueng-Cheng Yang ${ }^{5}$ and Tin Wee Tan²

Address: ${ }^{1}$ Department of Chemistry and Biomolecular Sciences and ARC Centre of Excellence in Bioinformatics, Macquarie University, Sydney NSW 2109, Australia, ${ }^{2}$ Department of Biochemistry, Yong Loo Lin School of Medicine, National University of Singapore, 8 Medical Drive, Singapore 117597, ${ }^{3}$ Institute of Information Science, Academia Sinica, Nankang, Taipei, Taiwan, ROC, ${ }^{4}$ Department of Computer Science, National Tsing-Hua University, Hsinchu, Taiwan, ROC and ${ }^{5}$ Institute of Biomedical Informatics and Center for Systems and Synthetic Biology, National Yang-Ming University, Taiwan, ROC

Email: Shoba Ranganathan* - shoba.ranganathan@mq.edu.au; Wen-Lian Hsu - hsu@iis.sinica.edu.tw; Ueng-Cheng Yang - yang@ym.edu.tw; Tin Wee Tan - tinwee@bic.nus.edu.sg

* Corresponding author

from Asia Pacific Bioinformatics Network (APBioNet) Seventh International Conference on Bioinformatics (InCoB2008)

Taipei, Taiwan. 20-23 October 2008

Published: 12 December 2008

BMC Bioinformatics 2008, 9(SuppI I2):SI doi:I0.1 I86/I47I-2I05-9-SI2-SI

This article is available from: http://www.biomedcentral.com/|47|-2/05/9/SI2/SI

(C) 2008 Ranganathan et al; licensee BioMed Central Ltd.

This is an open access article distributed under the terms of the Creative Commons Attribution License (http://creativecommons.org/licenses/by/2.0), which permits unrestricted use, distribution, and reproduction in any medium, provided the original work is properly cited.

\begin{abstract}
The 2008 annual conference of the Asia Pacific Bioinformatics Network (APBioNet), Asia's oldest bioinformatics organisation set up in 1998, was organized as the $7^{\text {th }}$ International Conference on Bioinformatics (InCoB), jointly with the Bioinformatics and Systems Biology in Taiwan (BIT 2008) Conference, Oct. 20-23, 2008 at Taipei, Taiwan. Besides bringing together scientists from the field of bioinformatics in this region, $\ln C o B$ is actively involving researchers from the area of systems biology, to facilitate greater synergy between these two groups. Marking the $10^{\text {th }}$ Anniversary of APBioNet, this InCoB 2008 meeting followed on from a series of successful annual events in Bangkok (Thailand), Penang (Malaysia), Auckland (New Zealand), Busan (South Korea), New Delhi (India) and Hong Kong. Additionally, tutorials and the Workshop on Education in Bioinformatics and Computational Biology (WEBCB) immediately prior to the 20th Federation of Asian and Oceanian Biochemists and Molecular Biologists (FAOBMB) Taipei Conference provided ample opportunity for inducting mainstream biochemists and molecular biologists from the region into a greater level of awareness of the importance of bioinformatics in their craft. In this editorial, we provide a brief overview of the peer-reviewed manuscripts accepted for publication herein, grouped into thematic areas. As the regional research expertise in bioinformatics matures, the papers fall into thematic areas, illustrating the specific contributions made by APBioNet to global bioinformatics efforts.
\end{abstract}

\section{Historical notes}

Established in 1998 [1], the Asia-Pacific Bioinformatics Network (APBioNet, [2-4]) strives to bring together scien- tists from diverse disciplines, working together to advance the frontiers of bioinformatics. Following annual meetings held at the Pacific Symposium of Biocomputing 
(1998-2001), APBioNet executive committee members assisted in organizing InCoB2002 (the International Conference on Bioinformatics, 2002) in Bangkok and adopted this meeting as their annual conference. Since then, InCoB meetings have been held in Penang, Malaysia (2003); Auckland, New Zealand (2004); Busan, South Korea (2005), New Delhi, India (2006) and Hong Kong/ Hanoi (by videocasting) (2007), with InCoB2008 at Taipei, Taiwan [5], with attendance rising to the highest ever (over 1,000) in Delhi.

Pursuant to APBionet's initial efforts of the BioMirrors initiative, in collaboration with the Asia Pacific Advanced Network (APAN), [6] to develop the network infrastructure for maintaining bioinformatics databases and computational resources, Sangket et al. [7] developed a BitTorrent-based Peer-to-Peer (btP2P) file distribution model for automatic synchronization and distribution of large amounts of biological data suitable for developing countries which are connected with developed countries via the TransEurasia Information Network TEIN2 initiative. To facilitate replication of bioinformatics resource nodes, APBioNet was awarded a grant by the International Development Research Centre of Canada (IDRC) to build a user-friendly package APBioBox (2003) with Globus grid software for fast deployment of grid computing for bioinformatics applications and subsequently collaborated with Sun Microsystems' Asia Pacific Science and Technology Center (APSTC) to co-develop the BioCluster Grid on the Solaris platform. It facilitated the development of a Knoppix LiveCD of bioinformatics applications called APBioKnoppix (2004), and subsequently in conjunction with the ASEAN SubCommittee on Biotechnology and the National University of Singapore's Bioinformatics Centre, the BioSlax Unix bioinformatics package of LiveCD and bioinformatics on a thumb drive (2006) was developed. More recently, this was extended to a VMware virtual machine version (for Windows and Mac) and a BioSlax Server Version for rapid (5 min) deployment of a complete bioinformatics computational resource for a teaching or research laboratory with a 1 Terabyte USB external hard disk of BioMirrors and BLAST datasets (see http://www.bioslax.com), test-deployed in Vietnam and elsewhere in 2007. These activities represent APBioNet's growing efforts at contributing to the software development scene for bioinformatics, instead of being solely a passive recipient of technology.

While constantly striving to establish better access to data and analysis tools, APBioNet's priorities also extended to education and training of the life science community, with active participation in initiatives such as the $S^{*}$ Life Science Informatics Alliance [8] and Workshops on Education in Bioinformatics (WEB, initiated by SR) [9], to meld bioinformatics with mainstream life science research. Outreach training workshops and courses expanded to diverse locations in Asia were facilitated by sponsorships from FAOBMB, IUBMB and UNESCO, and this has resulted in a much greater awareness and interest amongst regional scientists in the various aspects of bioinformatics. In recent years the Asia Pacific region has shown the fastest growth in bioinformatics publications (D. Seaton, Elsevier, personal communication), and has hosted several reputable international conferences, with the past year alone supporting the Genome Informatics Workshop (GIW) in Singapore [10], the Asia Pacific Bioinformatics Conference (APBC) in Hong Kong and the Research in Computational Molecular Biology (RECOMB) Conference also in Singapore [11], culminating in InCoB2008 in Taipei. This trend perhaps foreshadows the location of the largest bioinformatics global meeting, the Intelligent Systems in Molecular Biology (ISMB) conference of the International Society for Computational Biology [12] in the Asia Pacific region in the near future.

Having reached an international peer-reviewed highimpact factor standard in journal publication, APBioNet embarked on further raising the standards for the region by publishing a dedicated BMC Bioinformatics supplement, starting in 2006 [13,14]. By 2008, manuscripts from APBioNet members have diversified and increased in quality and sophistication, and are now focussed on emerging bioinformatics research areas, including microRNAs; transcriptomes, SNPs and protein analyses as well as protein trafficking, systems biology, immunoinformatics, and biodiversity informatics, further considered below.

\section{Review policy}

Papers submitted to these proceedings were peer-reviewed by at least two reviewers, from the APBioNet/InCoB program committee members and external experts as required (listed in Additional File 1). InCoB2008 also provided multi-track submissions, with the inclusion of new sessions to present research highlights from recent publications and for showcasing technology developments. With tutorials aimed at introducing new concepts and advancing current practices, and a specialist workshop on bioinformatics education, InCoB2008 has achieved the status of a comprehensive international bioinformatics meeting in the Asia Pacific.

The aim of the editors was to select only the best papers from more than a dozen Asia Pacific countries. From the 47 full paper submissions, 33 were short listed for oral presentation. This supplement features 24 papers, reflecting an overall acceptance rate of $51 \%$, with seven more appearing in the online journal, Bioinformation [15]. The impressive extent of collaboration in bioinformatics 
research in the region is demonstrated by the permutations of co-authorship, variously involving papers from Australia, China, Hong Kong, India, Japan, Korea, Singapore, Taiwan, Thailand, UK and USA. A brief review of the various themes follows.

\section{MicroRNAs}

The discovery in 2004 of very short regulatory RNA segments, termed micro RNAs (miRNAs), has provoked intense research. Chang et al. [16] propose a novel relaxed variable kernel density estimator for predicting speciesspecific miRNA precursors, particularly suited to taxa distant from humans. The identification of false positives from such predictions is provided by Leung et al. [17], using a clustering approach. The biomolecules that these miRNAs bind to are predicted accurately by the support vector machine classification method of Yang et al. [18] while Tran et al. [19] explore the regulatory networks controlled by miRNAs.

\section{Transcriptome analysis}

The regulatory role of RNAs is dependent on their binding to specific proteins. Cheng et al. [20] present a novel position-specific scoring matrix method, of high sensitivity, for predicting the RNA-binding sites on proteins. Transcription regulation depends on the binding of several factors based on the presence of distinct signal regions. Lu et al. [21] propose a new algorithm to extract these transcription factor binding sites from a set of unaligned gene sequences. Eukaryotic genes are assembled from a set of exons, from which the intervening intronic elements have been spliced out. A new feature-based splice site detection method has been developed by Baten et al. [22] to accurately delineate the architecture of a multi-exonic gene.

\section{SNPs}

Single nucleotide polymorphisms (SNPs) are key elements in genomics research, importantly for correlating genetic variations with disease outcomes. VarDetect (Ngamphiw et al. [23]) is a new generation tool for the accurate detection of SNPs, while the functional implications of these variations can be assessed using FANS (Liu et al. [24]).

\section{Protein analysis}

Understanding protein interactions and networks can provide a deep insight into the functions of biological molecules generally. Lee et al. [25] have extended our knowledge of protein interactions to 18 species, using orthologous interacting pairs. Interactions between proteins are characterized by the surface features of their three dimensional structures. Chang et al. [26] propose a new approach to compute protein solvent accessibility, which will facilitate protein tertiary structure prediction from sequence. The conservation of functional residues in a protein or a domain is critical to maintaining biological function. The evolutionary forces acting on mutation sites can be appraised with WPSMaker (Lee et al. [27]). The functional significance of active site residues in an enzyme has been explored by in silico ligand docking by Gowthaman et al. [28], in the interests of developing anti-HIV drugs.

\section{Protein trafficking}

Proteins are functional only if they are transported to specific cellular compartments. Choo and Ranganathan [29] report the involvement of flanking residues from the mature protein moiety in a large-scale analysis of experimentally validation secretory signal peptides, while Mizuno et al. [30] have experimentally evaluated predicted peroxisomal targeting signals.

\section{Systems biology}

Temporal changes in the interactions and concentrations of biological molecules are critical to systems biology. Using model systems, Wu et al. [31] present a new approach to dynamic sensitivity analysis.

\section{Immunoinformatics}

As part of our quest to efficiently address healthcare issues, delving through the ever-increasing biomedical literature presents an immense challenge. Tsai et al [32] propose a new system to convert their machine-learning based text analysis output into standardized biomedical annotations. Yang et al. [33] have compiled a database linking genetic variations to disease, while Wang et al. [34] address the issue of immunoglobulin heavy chain diversity to allow understanding antibody maturation and antibody-based therapy design. Lim et al. [35] propose a new method for predicting the allergenicity of a protein from its sequence information while Lin et al. [36] have evaluated the efficacy of available major histocompatibility complex Class II peptide binding prediction servers for effective vaccine design. Hsu et al. [37] have combined candidate disease gene information for genetic studies with protein interaction networks to propose a pathogenetic disease model for schizophrenia.

\section{Biodiversity informatics}

The application of bioinformatics approaches to data from several other areas such as biodiversity and ecology is only logical [9]. In this area, Lim et al. [38] have developed an integrated Korean biodiversity and genetic information retrieval system to link species information with molecular data. Gaikwad et al. [39] have created a customary medicinal plant knowledge base, impressively integrating taxonomy, phytochemistry, biogeography and the biological activities of customary medicinal plant species with chemoinformatics, to facilitate the discovery of novel bioactive compounds. 


\section{Conclusion}

Considering the scope and depth of topics covered in this issue, Asia Pacific bioinformatics research has maintained its level of research achievement, previously set with high standards in 2006. This area is now acknowledged to be a core research discipline in our educational and research institutions. The efforts expended into setting up the bioinformatics resource infrastructure have started to yield dividends, with a number of papers bearing the names of graduate research students as first authors.

Further, bioinformatics is now considered essential to provide substantial support to genomics and health research, as reported by workers from Thailand [40]. Regional meetings such as the recent Second ASEANChina International Bioinformatics Workshop and the Third East Asia Bioinformation Network meeting [41], held in Singapore in 2008, focus on specific applications of local relevance and cooperation, such as bioinformatics in traditional medicines and in emerging infectious diseases. Such endeavours are essential in addressing issues on human resource development in bioinformatics and in combating global health threats. After 10 years, APBioNet is well positioned "to foster the growth of Bioinformatics and its allied disciplines in the Asia Pacific" [2], and to sustain a new generation of researchers in the 'post-omic' era.

\section{Competing interests}

The authors declare that they have no competing interests.

\section{Additional material}

\section{Additional File 1}

APBioNet InCoB2008 Program Committee members.

Click here for file

[http://www.biomedcentral.com/content/supplementary/14712105-9-S12-S1-S1.pdf]

\section{Acknowledgements}

We are grateful for the local organizers of the InCoB2008 conference, especially Ms Serena Lee, National Yang-Ming University, Taipei, Taiwan. We thank the referees for their dedication and effort in reviewing the manuscripts and Dr John Candlish for his editorial assistance. We also thank $B M C$ Bioinformatics for their support and encouragement.

This article has been published as part of BMC Bioinformatics Volume 9 Supplement 12, 2008: Asia Pacific Bioinformatics Network (APBioNet) Seventh International Conference on Bioinformatics (InCoB2008). The full contents of the supplement are available online at http://www.biomedcentral.com/ $|47|-2 \mid 05 / 9$ ? issue $=\mathrm{S} \mid 2$.

\section{References}

I. Sugawara H, Miyazaki S: Towards the Asia-Pacific Bioinformatics Network. Pac Symp Biocomput 1998:759-764.
2. The Asia-Pacific Bioinformatics Network [http://www.apbio net.org]

3. Miyano S, Ranganathan S: The Asia-Pacific Regional Perspective Bioinformatics. IEEE Intelligent Systems 200I, 16:19-6I.

4. Ranganathan S, Subbiah S, Tan TW: APBioNet: the Asia-Pacific regional consortium for bioinformatics. Appl Bioinformatics 2002, I: $101-105$

5. The Seventh International Conference on Bioinformatics (InCoB2008): National Yang-Ming University, Taipei, Taiwan [http://incob.apbionet.org/incob08/]

6. Gilbert D, Ugawa Y, Buchhorn M, Tan TW, Mizushima A, Kim H, Chon K, Weon S, Ma J, Ichiyanagi Y, Liou DM, Keretho S, Napis S: Bio-Mirror project for public bio-data distribution. Bioinformatics 2004, 20:3238-3240.

7. Sangket U, Phongdara A, Chotigeat W, Nathan D, Kim WY, Bhak J, Ngamphiw C, Tongsima S, Khan AM, Lin H, Tan TW: Automatic synchronization and distribution of biological databases and software over low-bandwidth networks among developing countries. Bioinformatics 2008, 24:299-30I.

8. Lim YP, Höög JO, Gardner P, S Ranganathan S, Andersson S, Subbiah S, Tan TW, Hide W, Weiss AS: The S-Star Trial Bioinformatics Course: An On-Line Learning Success. Biochem Mol Biol Edu 2003, $31: 20-23$.

9. Ranganathan S: Bioinformatics Education - Perspectives and Challenges. PLoS Comput Biol 2005, I:e52.

10. Genome Informatics Workshop 2007 [http:// www.comp.nus.edu.sg/ giw2007/]

11. The $12^{\text {th }}$ Annual International Conference on Research in Computational Molecular Biology (RECOMB2008) [http:// www.comp.nus.edu.sg/ recomb08/]

12. The International Society for Computational Biology [http:/ /www.iscb.org]

13. Ranganathan S, Tammi M, Gribskov M, Tan TW: Establishing bioinformatics research in the Asia Pacific. BMC Bioinformatics 2007, 7(Suppl 5):SI.

14. Ranganathan S, Gribskov M, Tan TW: Bioinformatics Research in the Asia Pacific - an update. BMC Bioinformatics 2007, 9(Suppl I):SI.

15. Bioinformation [http://www.bioinformation.net/]

16. Chang DTH, Wang CC, Chen JW: Using a kernel density estimation based classifier to predict species-specific microRNA precursors. BMC Bioinformatics 2008, 9(SuppI I 2):S2.

17. Leung WS, Lin MCM, Cheung DW, Yiu SM: Filtering of false positive microRNA candidates by a clustering-based approach. BMC Bioinformatics 2008, 9(SuppI I 2):S3.

18. Yang Y, Wang YP, Li KB: MiRTif: a support vector machinebased microRNA target interaction filter. BMC Bioinformatics 2008, 9(Suppl I 2):S4.

19. Tran DH, Satou K, Ho TB: Finding microRNA regulatory modules in human genome using rule induction. BMC Bioinformatics 2008, 9(SuppI I2):S5.

20. Cheng CW, Su ECY, Hwang JK, Sung TY, Hsu WL: Predicting RNA-binding sites of proteins using support vector machines and evolutionary information. BMC Bioinformatics 2008, 9(Suppl 12):S6.

21. Lu CC, Yuan WH, Chen TM: Extracting transcription factor binding sites from unaligned gene sequences with statistical models. BMC Bioinformatics 2008, 9(SuppI I 2):S7.

22. Baten AKMA, Halgamuge SK, Chang BCH: Fast splice site detection using information content and feature reduction. $B M C$ Bioinformatics 2008, 9(Suppl I2):S8.

23. Ngamphiw C, Supasak Kulawonganunchai S, Assawamakin A, Jenwitheesuk $E$, Tongsima S: VarDetect: a nucleotide sequence variation exploratory tool. BMC Bioinformatics 2008, 9(Suppl I 2):S9.

24. Liu CK, Chen YH, Tang CY, Chang SC, Lin YJ, Tsai MF, Chen YT, Yao A: FANS: Functional analysis of novel SNPs and mutations in human and mouse genomes. BMC Bioinformatics 2008, 9(Suppl 12):SIO.

25. Lee SA, Chan CH, Tsai CJ, Lai JM, Wang FS, Kao CY, Huang CYF: Ortholog-based protein-protein interaction prediction and its application to inter-species interactions. BMC Bioinformatics 2008, 9(Suppl I 2):SII.

26. Chang DTH, Huang HY, Syu YT, Wu CP: Real value prediction of protein solvent accessibility using enhanced PSSM features. BMC Bioinformatics 2008, 9(SuppI I 2):SI 2. 
27. Lee YS 3, Kim TH, Kang TW, Chung WH, Shin GS: WSPMaker: a web tool for calculating selection pressure in proteins and domains using window-sliding. BMC Bioinformatics 2008, 9(Suppl I2):SI3.

28. Gowthaman U, Jayakanthan M, Sundar D: Molecular docking studies of dithionitrobenzoic acid and its related compounds to protein disulfide isomerase: computational screening of inhibitors to HIV-I entry. BMC Bioinformatics 2008, 9(Suppl 12):SI4.

29. Choo KH, Ranganathan S: Flanking signal and mature peptide residues influence signal peptide cleavage. BMC Bioinformatics 2008, 9(SuppI I 2):SI5

30. Mizuno Y, Kurochkin IV, Herberth M, Okazaki Y, Schönbach C: Predicted mouse peroxisome-targeted proteins and their actual subcellular locations. BMC Bioinformatics 2008, 9(SuppI I2):SI6.

31. Wu WH, Wang FS, Chang MS: Dynamic sensitivity analysis of biological systems. BMC Bioinformatics 2008, 9(Suppl I2):SI7.

32. Tsai RTH, Dai HJ, Huang CH, Hsu WL: Semi-automatic conversion of BioProp semantic annotation to PASBio annotation. BMC Bioinformatics 2008, 9(Suppl I 2):SI8.

33. Yang JO, Hwang S, Oh J, Bhak J, Sohn TK: An integrated databasepipeline system for studying single nucleotide polymorphisms and diseases. BMC Bioinformatics 2008, 9(SuppI I2):SI9.

34. Wang X, Wu D, Zheng S, Sun J, Tao L, Li Y, Cao Z: Ab-origin: an enhanced tool to identify the sourcing gene segments in germline for rearranged antibodies. BMC Bioinformatics 2008, 9(Suppl I 2):S20.

35. Lim S], Tong JC, Chew FT, Tammi MT: The value of position-specific scoring matrices for assess-ment of protein allergenicity. BMC Bioinformatics 2008, 9(SuppI I2):S2I.

36. Lin HH, Zhang GL, Tongchusak S, Reinherz EL, Brusic V: Evaluation of MHC-II peptide binding prediction servers: applications for vaccine research. BMC Bioinformatics 2008, 9(SuppI I 2):S22.

37. Hsu PC, Yang UC, Shih KH, Liu CM, Liu YL, Hwu HG: A protein interaction-based model for schizophrenia study. BMC Bioinformatics 2008, 9(Suppl I2):S23.

38. Lim J, Bhak J, Oh HM, Kim CB, Park YH, Paek WK: An integrated Korean biodiversity and genetic information retrieval system. BMC Bioinformatics 2008, 9(SuppI I 2):S24.

39. Gaikwad J, Khanna V, Vemulpad S, Jamie J, Kohen J, Ranganathan S: CMKb: a web-based prototype for integrating Australian Aboriginal customary medicinal plant knowledge. BMC Bioinformatics 2008, 9(Suppl I 2):S25.

40. Tongsima W, Tongsima S, Palittapongarnpim P: Outlook on Thailand's Genomics and Computational Biology Research and Development. PLoS Comput Biol 2008, 4:el000II5.

41. The 3rd East Asia Bioinformation Network meeting, Singapore [http://eabn.apbionet.org/3eabn08/]

Publish with Bio Med Central and every scientist can read your work free of charge

"BioMed Central will be the most significant development for disseminating the results of biomedical research in our lifetime. "

Sir Paul Nurse, Cancer Research UK

Your research papers will be:

- available free of charge to the entire biomedical community

- peer reviewed and published immediately upon acceptance

- cited in PubMed and archived on PubMed Central

- yours - you keep the copyright
BioMedcentral 